\title{
Universiteit
}

Leiden

The Netherlands

\section{Enhancing the charge ordering temperature in thin films of} Pr0.5Ca0.5MnO3 by strain

Yang, Z.-Q.; Zhang, Y.; Aarts, J.; Wu, M.-Y.; Zandbergen, H.W.

\section{Citation}

Yang, Z. -Q., Zhang, Y., Aarts, J., Wu, M. -Y., \& Zandbergen, H. W. (2006). Enhancing the charge ordering temperature in thin films of Pr0.5Ca0.5MnO3 by strain. Applied Physics Letters, 88, 072507. doi:10.1063/1.2172715

Version: $\quad$ Not Applicable (or Unknown)

License: $\quad$ Leiden University Non-exclusive license

Downloaded from: https://hdl.handle.net/1887/44542

Note: To cite this publication please use the final published version (if applicable). 


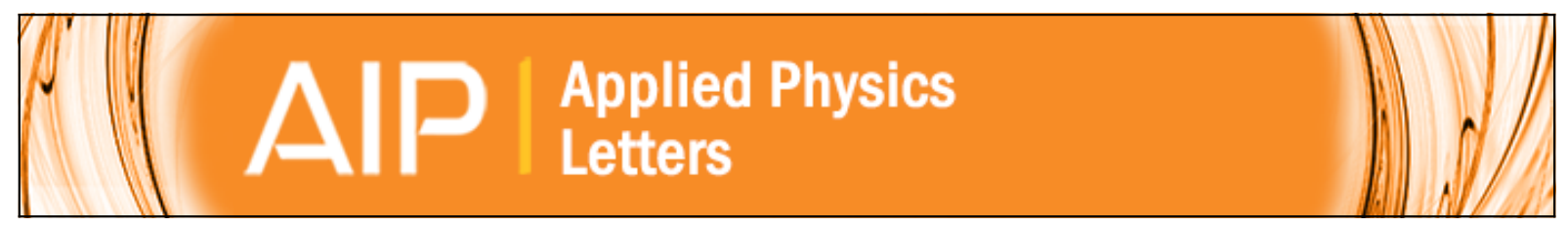

\section{Enhancing the charge ordering temperature in thin films of $\mathrm{Pr} 0.5 \mathrm{Ca} 0.5 \mathrm{MnO} 3$ by} strain

Z. Q. Yang, Y. Q. Zhang, J. Aarts, M.-Y. Wu, and H. W. Zandbergen

Citation: Applied Physics Letters 88, 072507 (2006); doi: 10.1063/1.2172715

View online: http://dx.doi.org/10.1063/1.2172715

View Table of Contents: http://scitation.aip.org/content/aip/journal/apl/88/7?ver=pdfcov

Published by the AIP Publishing

\section{Articles you may be interested in}

Influence of lattice strain on charge/orbital ordering and phase separation in $\operatorname{Pr} 0.7(\mathrm{Ca} 0.6 \mathrm{Sr} 0.4) 0.3 \mathrm{MnO} 3$ thin films

J. Appl. Phys. 115, 17D708 (2014); 10.1063/1.4863383

Defect-induced charge-order melting in thin films of $\operatorname{Pr} 0.5 \mathrm{Ca} 0.5 \mathrm{Mn} \mathrm{O} 3$

J. Appl. Phys. 101, 063919 (2007); 10.1063/1.2710341

Enhanced magnetoresistance in strain-free manganite network

Appl. Phys. Lett. 86, 062502 (2005); 10.1063/1.1861493

Magnetotransport properties in thin films of charge-ordered materials

J. Appl. Phys. 89, 8057 (2001); 10.1063/1.1372369

Microstructure and magnetic properties of strained La $0.7 \mathrm{Sr} 0.3 \mathrm{MnO} 3$ thin films

J. Appl. Phys. 88, 4257 (2000); 10.1063/1.1309040

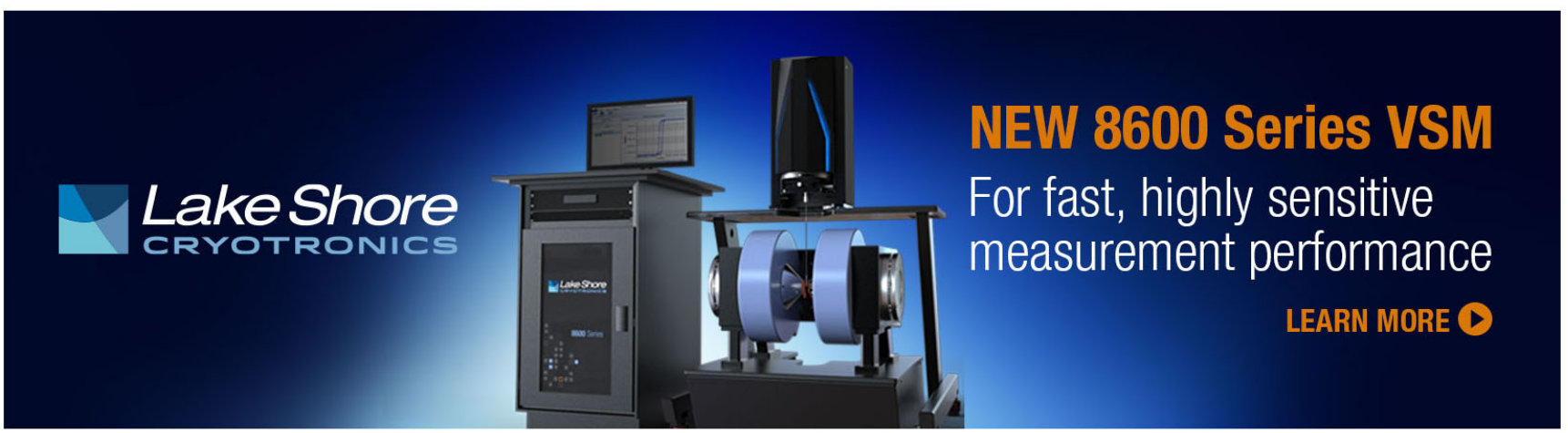




\title{
Enhancing the charge ordering temperature in thin films of $\mathrm{Pr}_{0.5} \mathrm{Ca}_{0.5} \mathrm{MnO}_{3}$ by strain
}

\author{
Z. Q. Yang, Y. Q. Zhang, ${ }^{\text {a) }}$ and J. Aarts \\ Kamerlingh Onnes Laboratory, Leiden University, P.O. Box 9504, 2300RA Leiden, The Netherlands
}

M.-Y. Wu and H. W. Zandbergen

Kavli Institute of Nanoscience, Delft University of Technology, Delft, The Netherlands

(Received 18 May 2005; accepted 13 January 2006; published online 17 February 2006)

\begin{abstract}
We report the effects of biaxial strain on the charge ordering temperature $T_{\text {co }}$ of the mixed-valent manganite perovskite oxide $\operatorname{Pr}_{0.5} \mathrm{Ca}_{0.5} \mathrm{MnO}_{3}$. Thin films were grown on $\mathrm{SrTiO}_{3}$, which has a $1.3 \%$ larger in-plane lattice parameter. Other substrates were used for comparison. Transport measurements combined with data from electron microscopy show that $T_{\text {co }}$ is considerably enhanced. At thicknesses of the order of $10 \mathrm{~nm}$, where the films are fully strained, $T_{\text {co }}$ is above $320 \mathrm{~K}$, more than $70 \mathrm{~K}$ above the bulk value of $250 \mathrm{~K}$, while around $50 \mathrm{~nm}$, where relaxation has set in, the enhancement is around $40 \mathrm{~K}$. The bulk value is only reached at a thickness of about 150 nm. (C) 2006 American Institute of Physics. [DOI: 10.1063/1.2172715]
\end{abstract}

Generally, the application of strain by growing a thin film on a nonmatched substrate can be used to alter the properties of a material. In particular in manganite perovskite oxides, which possess electrical and magnetic properties that are inherently sensitive to the structure of the material, strain engineering should be possible. Still, the effects of strain have not been very clear. For instance, in $\mathrm{La}_{0.67} \mathrm{Ba}_{0.33} \mathrm{MnO}_{3}$, which shows the well-known combined insulator-to-metal and paramagnetic-ferromagnetic transitions that lead to colossal magnetoresistance behavior, it has been shown that in-plane tensile strain by growth on $\mathrm{SrTiO}_{3}$ (STO) leads to a decrease of the ferromagnetic transition temperature by up to $40 \%{ }^{1}$ A similar conclusion was reached for $\mathrm{La}_{0.67} \mathrm{Ca}_{0.33} \mathrm{MnO}_{3}$ on STO. ${ }^{2}$ Clouding the issue, however, is the fact that the introduction of disorder leads to a similar decrease. Disorder is difficult to avoid, if only since one source for it is strain relaxation which already sets in above $10 \mathrm{~nm}$. If disorder is in competition with strain, the question arises whether strain can still significantly change the materials properties against disorder. We investigate this point by studying the behavior of the charge ordering temperature of thin films of $\operatorname{Pr}_{0.5} \mathrm{Ca}_{0.5} \mathrm{MnO}_{3}$ (PCMO) on different matching and nonmatching substrates. This choice of property and material requires some explanation.

PCMO is a perovskite oxide with the orthorhombic Pnma structure, where the $\mathrm{Mn}$ ions are encaged in cornersharing octahedra. The Mn-O-Mn skeleton is almost cubic, with a pseudocubic lattice parameter $a_{c}$, but small rotations of the octahedra lead to the orthorhombic structure, described by a unit cell $\left(a_{c} \sqrt{2}, 2 a_{c}, a_{c} \sqrt{2}\right)$. The almost cubic environment of the Mn-ions leads to a crystal field splitting of the $d$ states, with a triplet $t_{2 g}$ state (lower) and a doublet $e_{g}$ state (upper). Due to the $1: 1$ ratio of $\operatorname{Pr}^{3+}$ and $\mathrm{Ca}^{2+}$, there are equal amounts of $\mathrm{Mn}^{3+}$ ions and $\mathrm{Mn}^{4+}$ ions, which in the bulk undergo a transition into a checkerboard-type chargeordered state around $250 \mathrm{~K} .^{3,4}$ However, not only the charge orders. The degeneracy of the singly occupied $e_{g}$ state of the

\footnotetext{
${ }^{a)}$ Permanent address: Shenyang National Laboratory for Materials Science, Institute of Metal Research, Chinese Academy of Sciences, Shenyang, People's Republic of China.
}

$\mathrm{Mn}^{3+}$ ion is lifted by a Jahn-Teller distortion of the oxygen octahedron, which leads to a highly directional orbital state, pointing along one of the two Mn-O axes [the $(1,0,1)$ - and $(1,0,-1)$-directions $]$ in the $a-c$ plane of the crystal. Upon the occurrence of charge order (CO), orbital order (OO) occurs as well, with the lobes on subsequent $\mathrm{Mn}^{3+}$-ions alternating along the two Mn-O axes. ${ }^{5,6}$ The ordering phenomena have been well studied, and were shown to occur simultaneously in the bulk material at a temperature $T_{\text {coo }}$ of about $250 \mathrm{~K}$. We also mention that the magnetic moments on the Mn-ions order antiferromagnetically at $175 \mathrm{~K}$.

Important for possible strain effects in films is that at $T_{\text {coo }}$ the bulk lattice parameters change under influence of the Jahn-Teller distortion. The $a, c$-axes increase from $0.3820 \mathrm{~nm}$ to $0.3845 \mathrm{~nm}$, while the $b$-axis decreases from $0.3805 \mathrm{~nm}$ to $0.3745 \mathrm{~nm}$. $^{3}$ If a film is grown on a substrate with a larger lattice parameter such as STO $\left(a_{c}=0.391 \mathrm{~nm}\right)$, this may therefore lead to easier stabilization of the JahnTeller distortions and the concommittant $\mathrm{COO}$ order. What makes PCMO of particular interest is that it is already known what the effects of strained growth are on the so-called charge-order melting field; the insulating $\mathrm{CO}$ state is destroyed (melted) in a high magnetic field, since alignment of the Mn-moments leads to increased mobility of the $d$ electrons and metallic behavior. It was shown before that in very thin films (below $25 \mathrm{~nm}$ ) of PCMO on STO the melting fields are of the same order of magnitude as the bulk, but that they decrease quickly with increasing thickness. So, although an increase could have been expected on the same general argument of increased stability of the $\mathrm{COO}$ state, this was not found. Instead, the decrease (and the change in hysteretic behavior) was attributed to the increasing disorder by the relaxation of strain. ${ }^{8}$ Since it appears that disorder wins from strain in the case of $\mathrm{CO}$ melting, this leaves the question whether that is also the case for the charge order itself.

Determining $T_{\text {coo }}$ can be done in several different indirect ways, such as by measuring the temperature dependence of the resistance $R(T)$, which shows an upward kink, or of the magnetization $M(T)$, which shows a peak. ${ }^{9}$ In thin films, these signatures can be very weak. A direct way is to use an electron microscope in order to observe the charge order and 

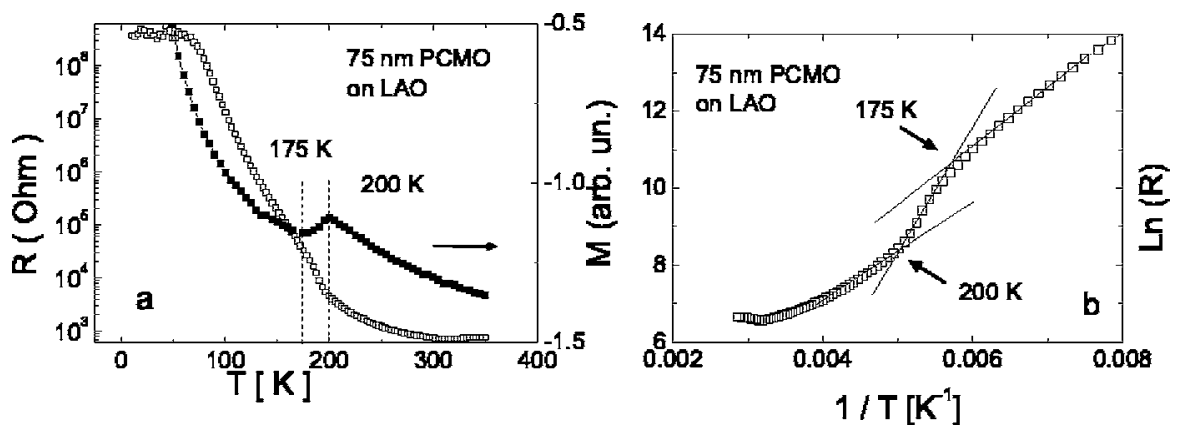

FIG. 1. (a) Resistance $R$ and magnetization $M$ as function of temperature $T$ for a film of $75 \mathrm{~nm}$ of PCMO on LAO. Dashed lines denote the temperatures of the maximum and minimum in $M$. (b) Plot of $\ln (\mathrm{R})$ vs $1 / T$. The line constructions show the three different regimes around $T_{\text {coo. }}$.

orbital order diffraction spots, and their disappearance as function of temperature. We present results from both $R(T)$ and electron diffraction (ED) for the determination of $T_{\text {coo }}$ of films of PCMO with different thickness under tensile strain on STO. We show that they yield the same results, and that $T_{\text {coo }}$ is significantly enhanced by strain. For comparison, we also present results for films on lattice-matched $\mathrm{SrLaGaO}_{4}$ (SLGO), and on $\mathrm{LaAlO}_{3}$ (LAO), with a smaller lattice parameter $\left(a_{c}=0.379 \mathrm{~nm}\right)$. All films were sputter-deposited from a ceramic target of nominally $\mathrm{Pr}_{0.5} \mathrm{Ca}_{0.5} \mathrm{MnO}_{3}$, in a pure oxygen atmosphere of $300 \mathrm{~Pa}$ with a substrate-source onaxis geometry, at a growth temperature of $840{ }^{\circ} \mathrm{C}$.

In order to illustrate the analysis used to extract $T_{\text {coo }}$ from the $R(T)$ data, Fig. 1(a) shows $R(T)$ as well as the magnetization $M(T)$ in a field of $0.5 \mathrm{~T}$ for a $75 \mathrm{~nm}$ thick film of PCMO on LAO. The sample both shows a clear peak in $M(T)$ at $200 \mathrm{~K}$ and an upward kink in the resistance at the same temperature. The features here are well distinguishable because the transition width $\Delta_{\text {tr }}$ is only $25 \mathrm{~K}$. In most samples they are considerably broader, and then $T_{\text {coo }}$ can better be determined from the plot of $\ln (R)$, versus $1 / T$, as shown in Fig. 1(b). The plot shows three regimes, one at high temperatures, a transition region where $R$ increases more strongly, and one at low temperatures. From comparison with the $M(T)$ data, $T_{\text {coo }}$ can be defined at the onset of the resistance increase, while $\Delta_{\text {tr }}$ is simply given by the width of the middle region. The plot also shows that $R(T)$ above $T_{\text {coo }}$ is not fully described by simple activated behavior of the form $\ln (R)=C_{0}-U_{a} /\left(k_{B} T\right)$, with $C_{0}$ a constant and $U_{a}$ an activation energy. Still, the drawn line gives a value for $U_{a}$ of $1300 \mathrm{~K}$, which is reasonable for the formation of small polarons, as is often assumed. ${ }^{10}$ Figure $2(\mathrm{a})$ shows $R(T)$ data for a PCMO films with a thickness of $80 \mathrm{~nm}$ on STO. Melting fields and lattice parameters of this film have been reported earlier, ${ }^{8}$ and they show that the film is still under strain, although relaxation has set in. Also shown in $R(T)$ for
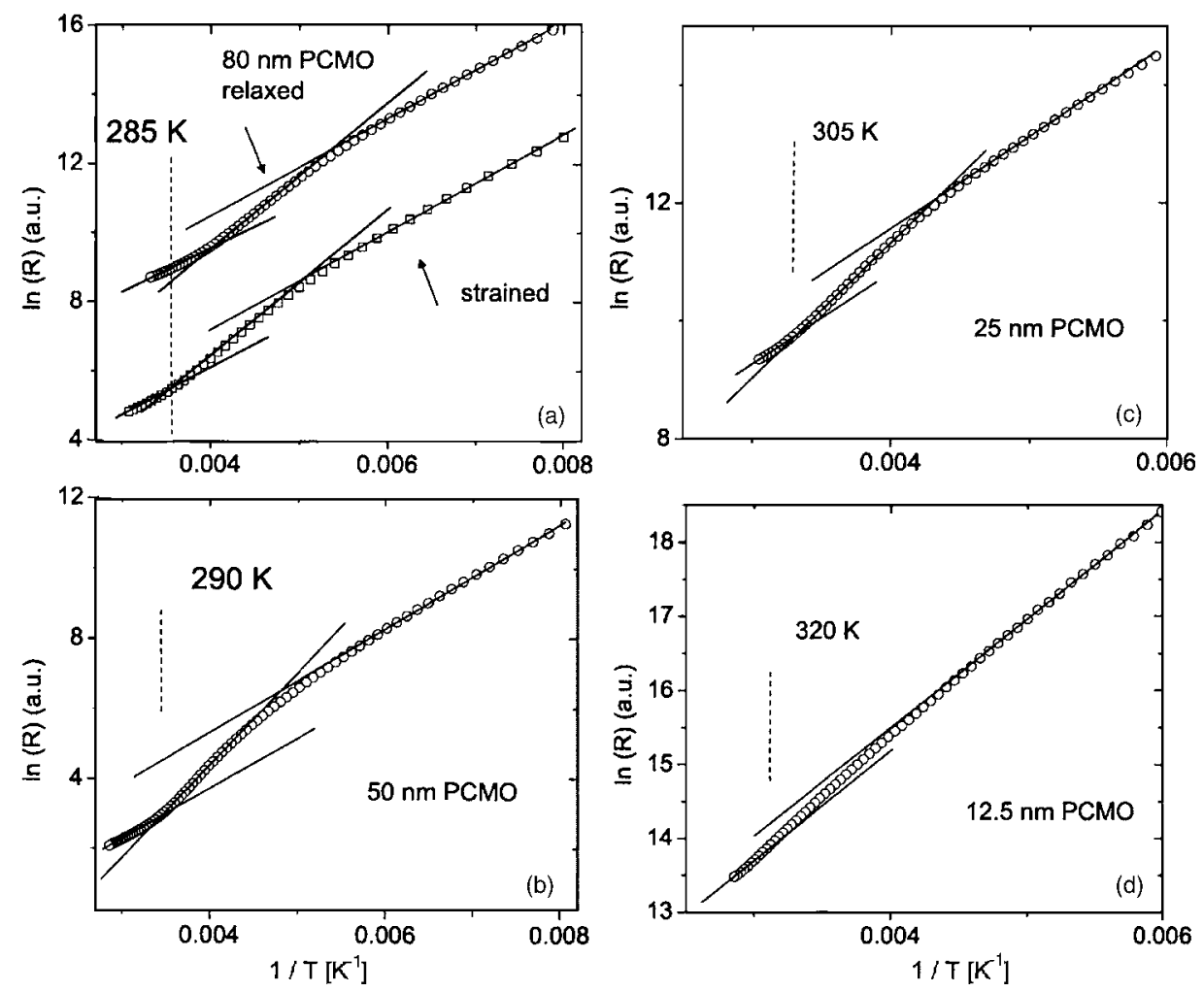

FIG. 2. Logarithm of the resistance $R$ vs inverse temperature $T$ for films of PCMO with various thickness on STO. Drawn lines indicate the transition regime, dashed lines the estimate for $T_{c \omega}$. (a) $80 \mathrm{~nm}$ strained and relaxed; the upper curve is shifted over 2 units. The dashed line is placed at $T_{c 00}$ for the strained film. (b) $50 \mathrm{~nm}$; (c) $25 \mathrm{~nm}$; (d) $12.5 \mathrm{~nm}$. 

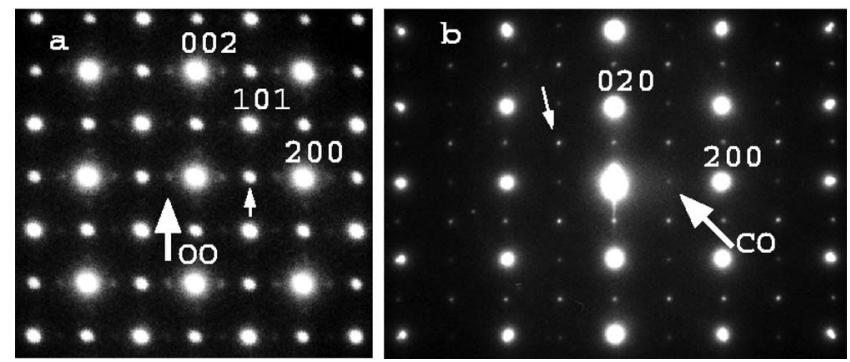

FIG. 3. (a) Electron diffraction pattern along the [010] zone axis for the film of $80 \mathrm{~nm}$ of PCMO on STO, taken at $95 \mathrm{~K}$. Indices mark Bragg peaks. The small arrow marks a kinematically forbidden (100) spot, the large arrow marked $\mathrm{OO}$ a half-order spot which is evidence for orbital order. (b) EDP along the [001] zone axis for the same film, taken at $295 \mathrm{~K}$. The small arrow marks a kinematically forbidden (110) spot, the large arrow marked CO a superstructure spot which is evidence for charge order.

an $80 \mathrm{~nm}$ film which has purposely been relaxed by postannealing for $5 \mathrm{~h}$ in $\mathrm{O}_{2}$. Both films show the three regimes, with $T_{\text {coo }}$ around $285 \mathrm{~K}$ for the strained layer and around $255 \mathrm{~K}$ for the relaxed layer. Values for $\Delta_{\text {tr }}$ are generally larger than found above, $75 \mathrm{~K}(80 \mathrm{~nm})$ and $80 \mathrm{~K}$ (relaxed $80 \mathrm{~nm})$, respectively. Values for $U_{a}$ are $1330 \mathrm{~K}(80 \mathrm{~nm})$ and $1100 \mathrm{~K}$ (relaxed $80 \mathrm{~nm}$ ). The data, and also those on a $50 \mathrm{~nm}$ thick film [Fig. 2(b)], suggest a modest increase of $T_{\text {coo }}$ at these thicknesses. For films of $25 \mathrm{~nm}$ or below [Fig. 2 (c) and 2(d)] $T_{\text {coo }}$ shifts to above $300 \mathrm{~K}$, and becomes difficult to define for the thinnest films of $12.5 \mathrm{~nm}$, since the maximum temperature of the measurement is $350 \mathrm{~K}$. Without supportive evidence, it would be difficult to make a strong case for an enhanced $T_{\text {coo }}$ but we can now compare these numbers to the data from ED. Figure 3(a) shows an electron diffraction pattern (EDP) taken on the $80 \mathrm{~nm}$ film along the $[010]_{P n m a}$ zone axis of the Pnma structure at $95 \mathrm{~K}$. Allowed Bragg peaks with index (002), (101), and (200) are marked. Along this zone axis, the extra reflections due to charge order would occur at the (001)- and (100)-positions, which are kinematically forbidden in the crystal structure. However, they can also appear due to multiple scattering, which is the case here, since they are found at all temperatures. More faint, but still clearly visible are spots in halforder positions, such as $\left(\frac{1}{2}, 0,0\right)$. They can only be explained by the occurrence of orbital order. Figure 3(b) shows an EDP taken on the $80 \mathrm{~nm}$ film along the $[001]_{\text {Pnma }}$ zone axis at $295 \mathrm{~K}$ (room temperature). Again, allowed Bragg peaks with index (020) and (200) are marked. Forbidden reflections of the (110)-type are also observed, which can be due to either multiple scattering or superposition of the 100 zone. Along this zone axis, however, the spots of the (100)-type are evidence for charge order. Orbital order along this axis cannot be observed. Figure 3(b) in itself is an important result, since it shows unequivocally that charge order exists far above the bulk value of $250 \mathrm{~K}$. Further experiments on the temperature dependence of the reflections were mostly performed on the orbital order spots. Because the films on STO grow with their $b$-axis out of the substrate plane, the $[010]_{P n m a}$ zone axis can be prepared in plane view, which is an easier procedure. Values of $T_{\text {coo }}$ were determined by measuring the temperature at which the relevant spots disappeared. All data

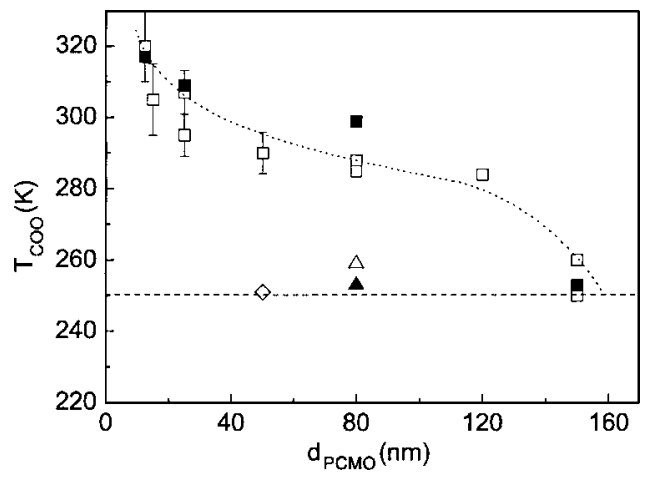

FIG. 4. Collection of values for $T_{\text {coo }}$ for films of PCMO with different thickness $d_{\mathrm{PCMO}}$ on different substrates. Open symbols are taken from resistance data, closed symbols from electron microscopy; ( $\square$ ) films on STO; $(\triangle)$ film relaxed by postanneal; $(\diamond)$ film on $\mathrm{SrLaGaO}_{4}$. The dotted line is a guide to the eye, the dashed line denoted the bulk value for $T_{\text {coo }}$.

are collected in Fig. 4. It can be seen that $T_{\text {coo }}$ for films on STO is almost always enhanced, while $T_{\text {coo }}$ for the film on matching SLGO has the bulk value. For films on LAO, $T_{\text {coo }}$ was generally found to be lower than the bulk value, which will be discussed elsewhere. The agreement between the $R(T)$ and the ED data is remarkably good. In particular, it is seen that both techniques show $T_{\text {coo }}$ to come back to the bulk value after strain relaxation. In the thinnest film of $12.5 \mathrm{~nm}$, $T_{\text {coo }}$ is at least $320 \mathrm{~K}$, which is $70 \mathrm{~K}$ above the bulk value. The behavior of $T_{\text {coo }}$ as a function of thickness appears to reflect the strain relaxation, which is known to set in above around $10 \mathrm{~nm}$. Above this thickness, $T_{\text {coo }}$ starts to come down, but is still enhanced over a large thickness range until the bulk value is reached around $150 \mathrm{~nm}$. From these direct observations, we feel safe to conclude that the tensile strain has indeed a strongly stabilizing effect on the occurrence of charge order in these mixed valent manganites.

This work is part of the research programme of the "Stichting voor Fundamenteel Onderzoek der Materie (FOM)," which is financially supported by the "Nederlandse Organisatie voor Wetenschappelijk Onderzoek (NWO)," Y.Q.Z. acknowledges a grant from the Dutch Academy of Science (K.N.A.W.).

${ }^{1}$ Y. Lu, J. Klein, C. Höfener, B. Wiedenhorst, J. B. Philipp, F. Herbstritt, A. Marx, L. Alff, and R. Gross, Phys. Rev. B 62, 15806 (2000).

${ }^{2}$ Z. Q. Yang, R. W. A. Hendrikx, J. Aarts, Y. L. Qin, and H. W. Zandbergen, Phys. Rev. B 70, 174111 (2004).

${ }^{3}$ Z. Jirák, S. Krupicka, Z. Simsa, M. Doulka, and S. Vratislav, J. Magn. Magn. Mater. 53, 153 (1985).

${ }^{4}$ Y. Tomioka, A. Asamitsu, H. Kuwahara, Y. Moritomo, and Y. Tokura, Phys. Rev. B 53, R1689 (1996).

${ }^{5}$ E. O. Wollan and W. C. Koehler, Phys. Rev. 100, 545 (1955).

${ }^{6}$ J. B. Goodenough, Phys. Rev. 100, 555 (1955).

${ }^{7}$ R. Kajimoto, H. Yoshizawa, Y. Tomioka, and Y. Tokura, Phys. Rev. B 63, 212407 (2001).

${ }^{8}$ Z. Q. Yang, R. W. A. Hendrikx, P. J. M. v. Bentum, and J. Aarts, Europhys. Lett. 58, 864 (2002); see also W. Prellier, A. M. Haghiri-Gosnet, B. Mercey, Ph. Lecoeur, M. Hervieu, Ch. Simon, and B. Raveau, Appl. Phys. Lett. 77, 1023 (2000) for a slightly different interpretation of the melting field behavior.

${ }^{9}$ Z. Jirák, F. Damay, M. Hervieu, C. Martin, B. Raveau, G. André, and F. Bourée, Phys. Rev. B 61, 1181 (2000).

${ }^{10}$ T. T. M. Palstra, A. P. Ramirez, S.-W. Cheong, B. R. Zegarski, P. Schiffer, and J. Zaanen, Phys. Rev. B 56, 5104 (1997). 